We hope to publish these results in more detail elsewhere at a later date.

E. Bradbury

C. Martin

British Cotton Industry Research Association,

Shirley Institute, Didsbury. June 20.

1 Pinoir, R., and Pouradier, J., C.R. Acad. Sci., Paris, 227, 190 (1948).

\section{Magnetic Properties, Internal Strains, and the Bauschinger Effect in Metals}

WILsoN $^{1}$ has recently reported that the intensity of magnetization of a high-carbon steel increases with plastic deformation of one kind, but that it suffers a recession after a relatively small additional plastic strain applied in a direction opposite to that originally used. I suggest that these results could be predicted from an analysis of the Bauschinger effect.

The conventional explanation of this effect ${ }^{2}$ accounts for the abrupt fall of resistance to deformation when the direction of the external stress is reversed (see diagram, $S$-curves). It is easy to see, however, that the simultaneous decrease of internal strain energy $E$ of the cold-worked metal can be only gradual, and that $E$ must reach a minimum $M$ before starting to rise again. Since work-hardening is never fully reversible, some residual strain energy $E_{r}$ remains latent in the metal.

This analysis implies that all energy-dependent properties affected in one manner during unidirectional deformation must be affected in the opposite manner on subsequent reversed straining. In fact, tensile elongation, recrystallization temperature, and sharpness of $\mathrm{X}$-ray diffraction rings and spots, do behave in the way expected ${ }^{3}$, and in a detailed report it will be shown that the Bauschinger effect is also accompanied by appreciable mechanical softening. The maximum decrease of Vickers hardness was 3-10 points in steels with $0 \cdot 07-0 \cdot 14$ per cent carbon, and up to 26 points in medium- and high-carbon steels with $0 \cdot 45-1 \cdot 15$ per cent carbon. Equivalent results were obtained with non-ferrous metals, and the amount of softening was consistent with the magnitude of the Bauschinger effect in each case.

Upon these findings, a general rule was formulated stating that work softening occurs whenever the method of cold working used tends to produce an internal stress system different from that left after the preceding treatment, irrespective of whether the latter was of mechanical or of thermal nature. Since the building-up of a new system of internal stresses must be preceded by a partial destruction of that existing previously, there is a transient decrease of $E$, demonstrated by softening.

The above rule immediately explains why the hardness of sorbitic and troostitic steels either remains constant $^{5,8}$ or even falls substantially ${ }^{4,7}$ during the early stages of cold working. The same apparently applies to magnetic properties ${ }^{1}$, and probably to density as well. Obviously, if 'back straining' is continued, the intensity of magnetization will also ultimately increase, but only after passing through a minimum, just like $E$ in the right-hand side of the diagram.

It seems, therefore, that Brinell or Vickers hardness tests provide a more convenient and sound basis for investigating internal stresses in metals than magnetic tests. Not only are they less cumbersome, but also they are more sensitive, since for reversed deformations varying from 4 to 8 per cent no difference whatever in intensity values was detected (ref. 1,

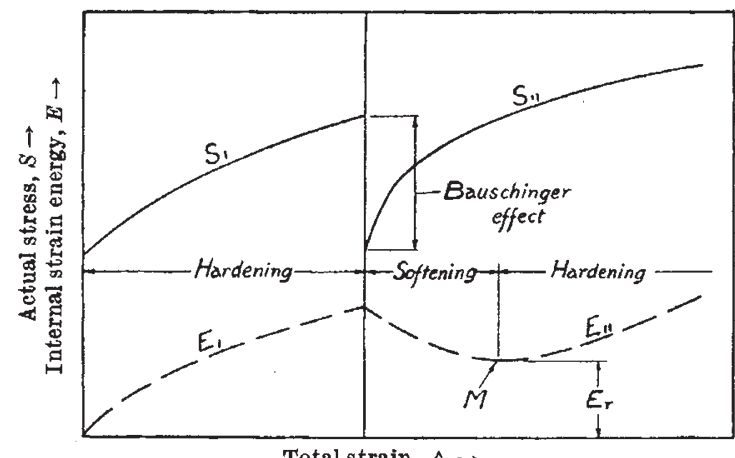

Total strain, $\Delta \rightarrow$

Stage 1, deformation in Stage 2, deformation in 8 one direction direction opposite to that of

Diagrammatic representation of changes of resistance to deformation $S$, and of internal strain energy $E$, associated with the Bauschinger effect. It is assumed that for $\triangle=0$, also $E=0$

curve 3). Furthermore, hardness tests are equally applicable to steels and non-ferrous metals, and are also sensitive to directional variations of properties. Finally, although the relations between hardness and stress values are not always clear, they are certainly simpler than those between magnetic properties and mechanical stresses.

Metallurgical Department,

\section{N. H. Polakowski}

University College, Swansea. June 12.

I Wilson, D. V., Nature, 167, 899 (1951).

${ }^{8}$ Barrett, C. S., "Strueture of Metals", 329 (MeGraw-Hill, New York, 1943).

s Burgers, W. G., “Handbuch der Metallphysik", 3, Pt. 2, 492 (review) (Akad. Verl. Becker and Frler, Leipzig, 1941)

'Polakowski, N. H., J. Iron and Steel Inst. (to be published).

- Andrew, J. H., and others, J. Iron and Steel Inst., 165, 145 (1950). - O'Neill, H., Proc. Inst. Mech. Eng., 151, 166 (1944).

${ }^{\prime}$ Armour, J. D., Trans. Amer. Soc. Steel Treat., 17, 521 (1930).

\section{Large Prime Numbers}

Fon about seventy-five years the largest known prime number has been $P=2^{127}-1$, identified as such by Edouard Lucas. It has remained the largest known in spite of many attempts to identify larger ones, although there have been conjectures and claims insufficiently substantiated or entirely unproved.

Recently we have prepared a routine for testing on the Edsac the primality of numbers of the form $k P+1$, with $P$ as defined above, and have found ten values of $k$ that give prime numbers. The largest of these gives the present (June 7) largest known prime, namely,

June 7. $934\left(2^{127}-1\right)+1$

\section{J. C. P. MUTLER}

D. J. WHEEIER

Note added in proof (October 8). Further work on the Edsac by Wheeler and myself has demonstrated the primality of

$$
978\left(2^{127}-1\right)+1
$$

and culminated in early July in the identification of the present largest known prime,

$$
180\left(2^{127}-1\right)^{2}+1 .
$$

Also, in early July, A. Ferrier, of France, using a desk machine, demonstrated the primality of

$$
\left(2^{148}+1\right) / 17 \text {, }
$$

which is the second largest known prime.

University Mathematical Laboratory,

$$
\text { J. C. P. Miller }
$$

